\title{
Articles
}

\section{Pregnancy and progression of diabetic nephropathy}

\author{
K. Rossing, P. Jacobsen, E.Hommel, E. Mathiesen, A. Svenningsen, P. Rossing, H-H. Parving \\ Steno Diabetes Center, Gentofte, Denmark
}

\section{Abstract}

Aims/hypothesis. Pregnancy could damage kidney function in diabetic nephropathy. We investigated the long-term impact of pregnancy on the progression of diabetic nephropathy.

Methods. Our observational follow-up study included all women patients with Type I (insulin-dependent) diabetes mellitus who developed diabetic nephropathy between 1970 and 1989 at Steno Diabetes Center $(n=93)$. Follow-up lasted 16 years (range 3-28) from the onset of diabetic nephropathy until death or the year 2000. A total of 26 women became pregnant after the onset of diabetic nephropathy (group A). The remaining 67 served as control subjects (group B). All patients received aggressive antihypertensive treatment (blood pressure goal $<140$ / $90 \mathrm{mmHg}$ ).

Results. The two groups were comparable at onset of diabetic nephropathy regarding blood pressure, albuminuria, s-cholesterol, smoking, retinopathy and screatinine (mean 79(SD 23) $\mu \mathrm{mol} / \mathrm{l})$. The slopes of $1 /$ s-creatinine $\left(1000 \cdot 1 \cdot \mu_{\mathrm{mol}^{-1}} \cdot\right.$ year $\left.^{-1}\right)$ during the whole observation period were $-0.39(0.40)$ compared with $-0.41(0.70)$ (group A vs B - NS). The slopes of $1 / \mathrm{s}$-creatinine before and after pregnancy were similar. Decline in creatinine clearance $(\mathrm{ml} / \mathrm{min} / \mathrm{yr})$ was 3.2 (3.4) compared with 3.2 (5.1) (group A vs B -NS). At the end of follow-up, 35\% (95\%-CI:17-53) of the pregnant women had died and 19\% (7-39) had reached end stage renal disease compared to $34 \%$ (23-45) and $24 \%(14-34)$ of the control subjects, respectively(NS). Group A and B had similar blood pressure levels during the whole observation period: 136(13)/83(7) vs 139 (14)/85(7) $\mathrm{mmHg}$ (NS).

Conclusion/interpretation. Pregnancy has no adverse long-term impact on kidney function and survival in Type I diabetic patients with well-preserved kidney function (normal serum creatinine) suffering from diabetic nephropathy. [Diabetologia (2002) 45: 36-41]

Keywords Diabetes mellitus, Type I diabetes, diabetic nephropathy, renal function, disease progression, pregnancy outcome, pregnancy in diabetes, longitudinally follow-up study.
Diabetic nephropathy is the most frequent cause of end stage renal disease (ESRD) in the western world [1]. Pregnancy causes major concern in women suffer-

Received: 20 June 2001 and in revised form: 21 September 2001

Corresponding author: K. Rossing, MD., Steno Diabetes Center, Niels Steensens Vej 2, DK-2820 Gentofte, Denmark, e-mail: krossing@dadlnet.dk

Abbreviations: ESRD, End-stage renal disease; GFR, glomenular filtration rate. ing from this complication particularly if serum creatinine is abnormally high because of the potential risk of a permanent deterioration in kidney function, greater risk of obstetric complications and congenital birth defects $[2,3]$. Consequently, many diabetologists (including those at the Steno Diabetes Center), nephrologists, and obstetricians advise their patients with diabetic nephropathy to avoid pregnancy. Two reviews dealing with the influence of pregnancy on the course of diabetic nephropathy indicated that pregnancy in itself does not accelerate the progression to end-stage renal disease $[4,5]$. However, previ- 
ously published studies did not simultaneously follow a control group of non-pregnant women with diabetic nephropathy, thus precluding the possibility of a valid assessment of the long-term outcome [6-11].

Therefore, our case-control study aimed to evaluate the long-term impact of pregnancy on the rate of decline in kidney function and on maternal survival in a consecutive group of patients with Type I (insulin-dependent) diabetes mellitus with diabetic nephropathy followed at one major diabetic centre in Denmark.

\section{Subjects and methods}

Patients. All women patients with Type I diabetes who developed diabetic nephropathy between 1970 and 1989 at Steno Diabetes Center and had at least 3 years of follow-up $(n=93)$ were enrolled in our study, irrespective of serum creatinine values (Table 1). Patients were followed from onset of diabetic nephropathy to death or to the year 2000 and no women were lost to follow-up.

Patients were divided into two groups according to whether or not they became pregnant after the onset of nephropathy. In the pregnant group (group A), we included all women with at least 20 weeks of gestation after the onset of diabetic nephropathy $(n=26)$, irrespective of serum creatinine values at onset of the pregnancy. The remaining 67 patients served as the control group (group B).

Diabetic nephropathy was diagnosed clinically if the following criteria were fulfilled: persistent albuminuria of more than $300 \mathrm{mg} / 24 \mathrm{~h}$ in at least two out of three consecutive 24-h urine collections, presence of diabetic retinopathy and the absence of any clinical or laboratory evidence of other kidney or renal tract disease [1]. The nephropathy diagnosis was always established before and never during pregnancy.

All patients had been dependent on insulin from the time of diagnosis, and all received at least two daily injections of highly purified porcine insulin during the 1970ties, increasing from two to 4 daily injections of human insulin during the following decades. Patients were treated with a diabetic diet without sodium or protein restriction.

A step-care approach for antihypertensive treatment was used. Before 1991 it included selective betablockers, diuretics and vasodilators. After 1991, it included angiotensin converting enzyme inhibitors, diuretics, calcium channel blockers (mainly dihydropyridines) and betablockers. During the whole follow-up period, we tried to keep blood pressure below 140/ $90 \mathrm{mmHg}$. During pregnancy hypertension was treated with either methyldopa or betablockers in combination with diuretics whereas ACE inhibitors were withdrawn. Patients were classified as taking a class of antihypertensive agent if the drug was prescribed for more than $50 \%$ of the follow-up time.

All physicians at the Steno Diabetes Center were engaged in the pre-pregnancy counselling. In general women suffering from diabetic nephropathy irrespective of their serum creatinine concentration were advised not to become pregnant. Pregnant patients were controlled according to a general set up for pregnant women at our hospital and the Department of Obstetrics at the University of Copenhagen. Patients were seen every two to three weeks at these two departments. Nonpregnant patients were seen regularly in our outpatient clinic approximately three to four times a year.

The local ethical committee approved the experiment's design and all patients gave their informed consent.
Procedures. Arterial blood pressure was measured twice after 5 to 10 min rest in a sitting position with a clinical sphygmomanometer. Diastolic blood pressure was taken at Korotkow 5. Hypertension was established if at least three measurements were equal to or higher than 140/90 $\mathrm{mmHg}$.

Albuminuria was determined from a 24-h urine collection carried out in relation to a visit to the outpatient clinic. Albuminuria was quantified initially by RIA technique [12] and later on by ELISA [13].

Serum creatinine was measured at least every year using a reaction rate kinetic technique eliminating pseudo creatinine (upper limit of normal in women $100 \mu \mathrm{mol} / \mathrm{l}$ ) [14]. The rate of decline in creatinine clearance for the entire follow-up period was estimated in the two groups using the Cockroft and Gault formula, which has been validated in diabetes [15]. End stage renal disease was diagnosed if the patients had a serum creatinine above $500 \mu \mathrm{mol} / \mathrm{l}$ or if the patients needed dialysis or had a kidney transplantation.

An annual measurement of glomerular filtration rate (GFR) was introduced in 1984 [16]. Consequently, only a subset $(n=59)$ of the 93 patients included in the study had this examination.

Haemoglobin $\mathrm{A}_{1 \mathrm{c}}\left(\mathrm{HbA}_{1 \mathrm{c}}\right)$ was measured from 1984 on high performance liquid chromatography (BIORAD/DIMAT, Richmond, Calif., USA, normal range 4.3 to $6.2 \%$ ) or by isoelectric focusing method (normal range $4.1-6.1 \%$ ).

Diabetic retinopathy was assessed either by direct ophthalmoscopy or fundus photo. Information on smoking was obtained from the medical records.

Fetal outcome, gestational age birth weight, malformations, and neonatal death were recorded. Cause of death was obtained from the death certificate. An independent observer without knowledge of which group the patient belonged to received all the death certificates and recorded the primary cause of death.

Statistical methods. Data are expressed as mean (SD) for normally distributed, geometric mean $(95 \%-\mathrm{Cl})$ for lognormal distributed data and median (range) for non-normally distributed data. The change in kidney function during follow-up was evaluated by linear regression analysis on reciprocal serum creatinine values, creatinine clearances and GFR determination. Baseline data, the change in kidney function and mean arterial blood pressure, albuminuria and $\mathrm{HbA}_{1 \mathrm{c}}$ during follow-up were compared by two sample Student's $t$ test. The cumulative incidences of doubling in serum creatinine, ESRD and mortality were calculated for one year intervals with a life-table method and the two groups were compared by the Logrank test.

Linear multivariate regression analysis with backward selection was performed with a decline in kidney function as a dependent variable and with or without pregnancy, arterial blood pressure, albuminuria and $\mathrm{HbA}_{1 \mathrm{c}}$ during follow-up as independent variables. All calculations were performed using SPSS 10.0 for Windows (SPSS, Chicago, Ill., USA). A $p$ value of less than 0.05 (two-sided) was considered to be statistically significant.

\section{Results}

The demographic and clinical data at onset of diabetic nephropathy in 93 women with Type I diabetes is shown in Table 1 . A total of 26 women became pregnant during follow-up (group A) and the remaining 
Table 1. Demographical and clinical data at onset of diabetic nephropathy in women with Type I diabetes

\begin{tabular}{|c|c|c|c|}
\hline & $\begin{array}{l}\text { Non-pregnant } \\
(n=67)\end{array}$ & $\begin{array}{l}\text { Pregnant } \\
(n=26)\end{array}$ & $p$ \\
\hline Age (years) & $27(7)$ & $24(4)$ & $<0.01$ \\
\hline Onset of diabetes (year) ${ }^{\mathrm{a}}$ & $1963(1937-1979)$ & $1968(1946-1979)$ & NS \\
\hline Diabetic duration (years) & $18(7)$ & $14(4)$ & 0.03 \\
\hline Systolic blood pressure (mmHg) & $133(14)$ & $128(11)$ & NS \\
\hline Diastolic blood pressure (mmHg) & $88(10)$ & $82(9)$ & NS \\
\hline \multicolumn{4}{|l|}{ Retinopathy (\%) } \\
\hline Non-proliferative & 45 & 50 & NS \\
\hline Proliferative & 55 & 50 & NS \\
\hline Patients with known cardiovascular disorder $(n)$ & 1 & 0 & NS \\
\hline S-creatinine $(\mu \mathrm{mol} / 1)$ & $79(23)$ & $79(23)$ & NS \\
\hline S-cholesterol (mmol/l) & $6.2(1.5)$ & $5.2(1.0)$ & NS \\
\hline
\end{tabular}

Values are mean (SD) ${ }^{a}$ median (range)

beometrical mean $(95 \%-C I)$

67 women served as control subjects (group B). In group A, five women became pregnant twice giving a total of 31 deliveries. The two groups were comparable except for a shorter duration of diabetes and a younger age at onset of nephropathy in group A. The pregnant group was followed for a median of 4 (1-17) years before pregnancy and 10(3-22) years after pregnancy (the latest if several). At onset of pregnancy, the median value of serum creatinine was 82 (range 42-148) $\mu \mathrm{mol} / \mathrm{l}$ and only three women had values above the upper normal limit of $100 \mu \mathrm{mol} / \mathrm{l}$. At onset of pregnancy, arterial blood pressure was $130(10) / 83(7) \mathrm{mmHg}$ and albuminuria 520(470-534) $\mathrm{mg} / 24 \mathrm{~h}$.

A total of $83(90 \%)$ patients were treated with antihypertensive medication during the whole or the major part of the follow-up period. Monotherapy was given to $43 \%$ of the patients, $30 \%$ received 2 agents, $15 \%$ received 3 agents and $2 \%$ were treated with 4 or more antihypertensive drugs.

There was no difference in the percentage of patients who received an ACE inhibitor in the two groups ( $44 \%$ in group A vs $46 \%$ in group B (NS)).

During follow-up, no significant difference was found between the pregnant group and the non-pregnant group regarding arterial blood pressure, albuminuria, $\mathrm{HbA}_{1 \mathrm{c}}$ and in any of the kidney endpoints: the slope of $1 /$ serum creatinine, decline in estimated creatinine clearance, doubling of serum creatinine, and end stage renal disease (Fig. 1, Table 2).

In the pregnant group, the slopes of $1 / \mathrm{s}$-creatinine both during the entire follow-up period (before and after pregnancy) and in the post partum period were similar to control subjects (Table 2). The corresponding mean differences $(95 \%-\mathrm{CI})$ (pregnant vs nonpregnant) were: $0.03(-0.32$ to 0.27$) 1000 \cdot 1 \cdot \mu \mathrm{mol}^{-1}$ - year ${ }^{-1}$ (entire follow-up) and 0.09 (-0.40 to 0.21$)$ $1000 \cdot 1 / \mu \mathrm{mol} / \mathrm{yr}$ (post partum).
In the subgroup of women in the pregnant group with at least three years of follow-up both before and after pregnancy $(n=17)$, the slope of $1 /$ s-creatinine $\left(1000 \cdot 1 \cdot \mathrm{mmol}^{-1} \cdot\right.$ year $\left.^{-1}\right)$ before pregnancy was $-0.13(0.86)$ compared to $-0.24(0.50)$ after pregnancy (mean difference (95\%-CI) 0.11 (-0.44 to 0.67$)$ ) (NS).

In the subgroup of patients where GFR determinations were carried out, we found a rate of decline in GFR during the whole follow-up period of 2.2 (2.1) $\mathrm{ml} / \mathrm{min} / \mathrm{yr}$ in the pregnant group $(n=17) \mathrm{com}$ pared to $3.6(3.8) \mathrm{ml} / \mathrm{min} / \mathrm{yr}(n=42)$ in the control group (mean difference (95\%-CI) 1.3 (0.6 to 1.3$)$ ) NS. These subgroups of patients were similar with regard to glomerular filtration rate, arterial blood pressure and albuminuria at baseline.

At the end of follow-up 5 women (19\% (95\%CI:7-39)) in the pregnant group had reached end stage renal disease compared to $16(24 \%(14-34))$ in the non-pregnant group (NS). Of the patients who reached end stage renal disease all five patients (100\% (46-100)) in the pregnant group died later on in life compared to eight out of 16 in the control

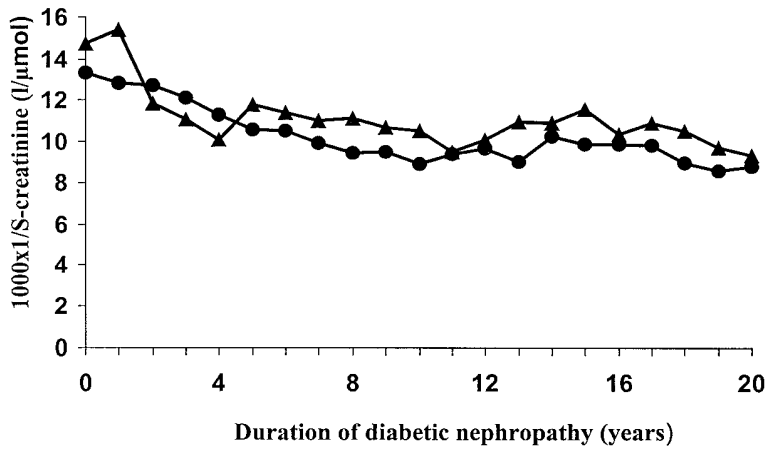

Fig. 1. Mean values of $1 / \mathrm{s}$-creatinine according to duration of diabetic nephropathy in women with Type I diabetes. Pregnant group ( $\mathbf{\Delta})$ and non-pregnant group ( $\mathbf{O})$ 
Table 2. Arterial blood pressure, albuminuria, haemoglobin $\mathrm{A}_{1 \mathrm{c}}$ and renal endpoints during the whole follow-up period in women with Type I diabetes and diabetic nephropathy

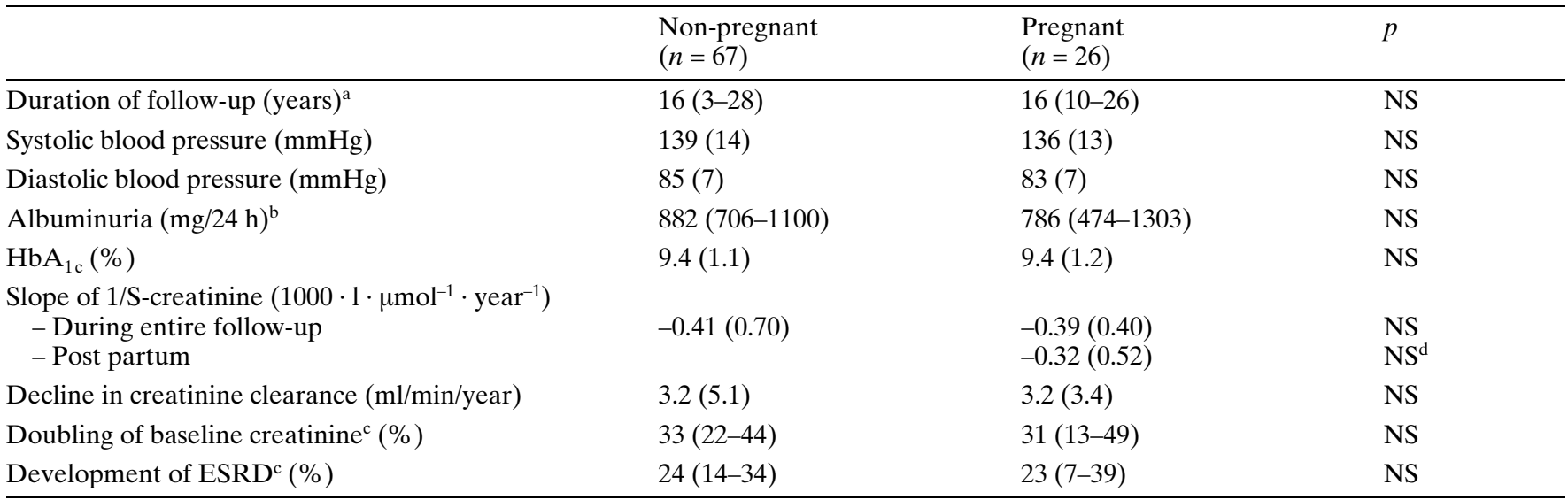

Values are mean (SD) ${ }^{\text {a }}$ median (range)

${ }^{\mathrm{b}}$ geometric mean $(95 \%-\mathrm{CI})$

group (50\% (28-72)) - (NS). The kidney transplantation rates were similar with $40 \%(5-85)$ in the pregnant group compared to $38 \%(15-65)$ in the nonpregnant group.

The main causes of death in both groups were due to cardiovascular and end stage renal disease (Table 3 ).

The cumulative incidence of the combined endpoint of end stage renal failure and death was nearly identical in the pregnant and in the non-pregnant group (Fig. 2). At the end of follow-up, 65\% (95\%CI: $47-83)$ in the pregnant group and $53 \%(41-65)$ in the non-pregnant group were still alive and without end stage renal disease.

In group A there were 31 deliveries during followup. In 12 of the deliveries a caesarean section was performed. Gestations lasted 37 (28-38) weeks (median (range)). The mean (SD) birth weight was 2535 (829) g. There were three neonatal deaths two due to respiratory distress and one due to myelomeningocele. There were two additional malformations present at birth including one with congenital dislocation of the hip and one with clubfoot. In group A there were four therapeutic abortions and four miscarriages during follow-up. In group B there were five therapeutic abortions and two miscarriages in seven different women. All abortions occurred before 12 weeks of gestation.

Table 3. Causes of death in women with Type I diabetes and diabetic nephropathy

\begin{tabular}{lcll}
\hline & $\begin{array}{l}\text { Non-pregnant } \\
(\mathrm{n}=67)\end{array}$ & $\begin{array}{l}\text { Pregnant } \\
(\mathrm{n}=26)\end{array}$ & $p$ \\
\hline Cardiovascular & $7(10 \%)$ & $4(15 \%)$ & \\
ESRD & $8(12 \%)$ & $5(19 \%)$ & \\
Accident & $1(2 \%)$ & $0(0 \%)$ & \\
Unknown & $4(6 \%)$ & $0(0 \%)$ & NS \\
Total & $23(34(23-45) \%)^{\mathrm{a}}$ & $9(35(17-53) \%)^{\mathrm{a}}$ & \\
\hline
\end{tabular}

a Percentage (95\%-CI)
${ }^{c}$ percentage $(95 \%$-C.I.)
d pregnant post partum vs non-pregnant during entire follow-
up

Before the development of diabetic nephropathy there were 18 pregnancies (five deliveries and 13 abortions) among the 26 women in group A and 35 (26 deliveries and eight abortions) among the 67 women in group B.

\section{Discussion}

In our long-term observational case-control study of all women patients with Type I diabetes $(n=94)$ developing diabetic nephropathy between 1970 and 1989 at the Steno Diabetes Center, we found no adverse impact of pregnancy on progression of diabetic nephropathy or survival in women with normal or near normal kidney function at conception. The actuarial kidney survival curves from onset of diabetic nephropathy to end stage renal disease or death for

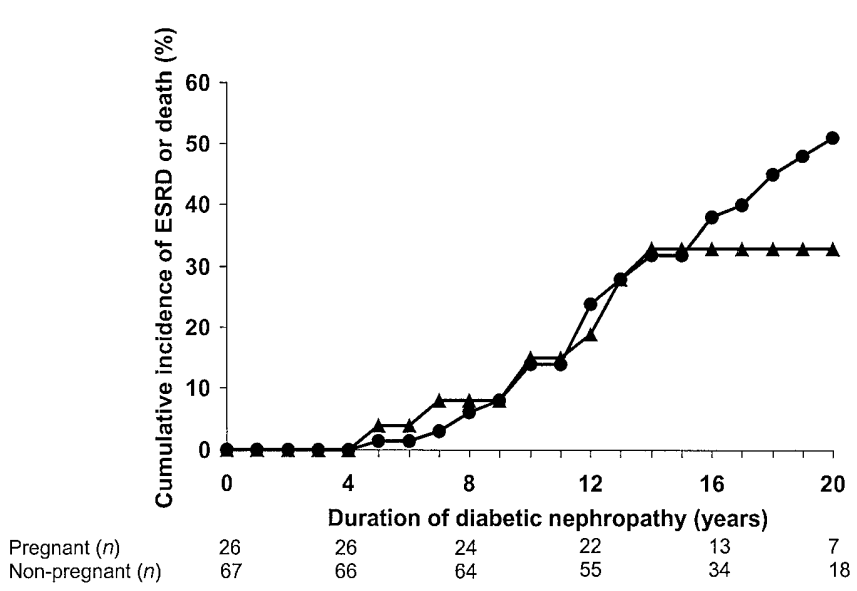

Fig. 2. Cumulative incidence of end stage renal disease or death vs years of diabetic nephropathy in the pregnant group $(\Delta)$ and the non-pregnant group (O). Numbers of patients at risk are indicated 
women who became pregnant did not differ from that observed in women who did not conceive after onset of renal disease. The patients benefited from improved prognosis as compared to the natural history of the diabetic nephropathy as discussed below [17-19]. The two groups were well matched at baseline and during follow-up regarding the major risk factors for losing filtration power i.e. arterial blood pressure, albuminuria, glycaemic control and lipids [20]. Aggressive antihypertensive treatment is thought to play a major role for the observed improvement in the progression of diabetic nephropathy.

The natural history (no-antihypertensive treatment) of diabetic nephropathy is characterised by a progressive rise in arterial blood pressure, proteinuria and a relentless decline in glomerular filtration rate (on average $10-15 \mathrm{ml} / \mathrm{min} /$ year) leading to end stage renal disease [17-19]. A median survival of 5-7 years after onset of proteinuria has been reported [21]. Our study in which aggressive antihypertensive treatment was applied revealed minimal changes in arterial blood pressure, a small rise in albuminuria, a major reduction in the rate of decline in glomerular filtration rate $(3.2 \mathrm{ml} / \mathrm{min} /$ year $)$ and improved survival (median $>18$ years). Those data are in agreement with the beneficial effect of antihypertensive treatment reported in many trials [22-25].

Previous studies of the influence of pregnancy on the progression of diabetic nephropathy have [6-8, 10] reported a much higher rate of decline in glomerular filtration rate $(10-15 \mathrm{ml} / \mathrm{min} /$ year $)$ compared to our findings [7, 9-11]. The risk of end stage renal disease was also higher than in our data. These disparities could well be explained by differences in antihypertensive treatment. Furthermore the numbers of patients in the previous studies were small and the follow-up time relatively short. The studies also lacked a simultaneously evaluated control group of women with diabetic nephropathy, making a valid conclusion impossible. In contrast, a study of a large cohort of non-diabetic patients over a long follow-up period demonstrates strong evidence, that pregnancy does not adversely affect the course of renal disease in women with various forms of non-diabetic glomerulopathies, provided that they have normal renal function at conception [26].

Studies on patients with reduced kidney function (above upper limit of normal serum creatinine) in diabetic and non-diabetic nephropathy have, however, suggested a permanent deterioration in renal function [8] [2] with an increased risk of end stage renal disease and death within a few years after delivery.

In our study, only three women had abnormally high values of serum creatinine at the onset of the pregnancy. Consequently, a separate analysis of the impact of pregnancy in women with impaired renal function could not be done. The low number of women with raised serum creatinine at conception was due to a general policy to advise women with nephropathy not to become pregnant, particularly if their kidney function was reduced. On purpose we included all women who became pregnant irrespective of serum creatinine at onset of pregnancy to avoid any selection bias in relation to the control group. A secondary analysis in which the three women with higher serum creatinine than normal at onset of pregnancy were excluded, did not change the conclusions of our study.

Though the prognosis might not be affected by pregnancy, at least in women with preserved kidney function as suggested in our study, morbidity and mortality are high in patients with diabetic nephropathy. Ten years after the last delivery approximately one third of the women had developed end-stage renal disease or died. Five patients in the pregnant group developed ESRD after respectively 5, 7, 12, 13 and 22 years of diabetic nephropathy, corresponding to $2,6,7,9$ and 20 years after pregnancy. Nine patients in the pregnant group died and the median (range) age of their children when the patients died was 9 (3-17) years.

In this study we found a very high abortion rate, especially before the development of diabetic nephropathy in the pregnant women. An abortion rate of approximately $40 \%$ has been found in a previous population-based survey of self-reported abortions in Danish women with Type I diabetes [27]. The generally high abortion rate found in our study could be a chance finding or perhaps be due to poor metabolic control [28] or the existence of microangiopathy [29].

The low birth weight, short gestational age and high percentage of neonatal deaths, congenital abnormalities and caesarean sections found in our and previous studies [4-11] reflect the many difficulties associated with these vulnerable pregnancies. It is also well documented that pregnancy in patients with nephropathy induces short-term blood pressure increases and greater proteinuria $[6,30]$. The predictive power of such abnormalities in our study could not be evaluated because data from our records and from the obstetrical records were incomplete. However, patients should now be informed that pregnancy leaves the long-term prognosis of diabetic nephropathy unaffected provided care is appropriate and kidney function is well-preserved.

In conclusion, pregnancy has no adverse long-term impact on kidney function and survival in Type I diabetic patients with nephropathy and well-preserved kidney function at the onset of pregnancy. Aggressive antihypertensive treatment is believed to improve the prognosis in diabetic nephropathy.

Acknowledgements. The Danish Diabetes Association supported the study. We would like to thank Dr R.W. Bilous for scientific and linguistic comments. 


\section{References}

1. Parving H-H, Østerby R, Ritz E (2000) Diabetic nephropathy. In: Brenner BM, Levine S (eds) The Kidney, 6th edn vol 2. WB Saunders, Philadelphia, Pennsylvania, pp 1731-1773

2. Prudy L, Hantsch C, Molitch M et al. (1996) Effect of pregnancy on renal function in patients with moderate-to-serve diabetic renal insufficiency. Diabetes Care 19: 1067-1067

3. Mackie A, Doddridge M, Gamsu H, Brudenell J, Nicolaides K, Drury P (1996) Outcome of pregnancy in patients with insulin-dependent diabetes mellitus and nephropathy with moderate renal impairment. Diabet Med 13: 90-96

4. Reece E, Leguizamon G, Homko C (1998) Pregnancy performance and outcomes associated with diabetic nephropathy. Am J Perinatol 15: 413-421

5. Leguizamon G, Reece E (2000) Effect of medical therapy on progressive nephropathy: influence of pregnancy, diabetes and hypertension. J Matern Fetal Med 9: 70-78

6. Kimmerle R, Zass R-P, Cupisti S et al. (1995) Pregnancies in women with diabetic nephropathy: long-term outcome for mother and child. Diabetologia 38: 227-235

7. Kitzmiller J, Combs A (1996) Diabetic nephropathy and pregnancy. Obstet Gynecol Clin North Am 23: 173-173

8. Biesenbach G, Stöger H, Zazgornik J (1992) Influence of pregnancy on progression of diabetic nephropathy and subsequent requirement of renal replacement therapy in female type I diabetic patients with impaired renal function. Nephrol Dial Transplant 7: 105-109

9. Miodovnik M, Rosenn B, Khoury J, Grigsby J, Siddiqi T (1996) Does pregnancy increase the risk for development and progression of diabetic nephropathy? Am J Obstet Gynecol 174: 1180-1191

10. Gordon M, Landon M, Samuels P, Hissrich S, Gabbe S (1996) Perinatal outcome and long-term follow-up associated with modern management of diabetic nephropathy. Obstet Gynecol 87: 401-409

11. Reece E, Winn H, Hayslett J, Coulehan J, Wan M, Hobbins J (1990) Does pregnancy alter the rate of progression of diabetic nephropathy? Am J Perinatol 7: 193-193

12. Miles D, Mogensen C, Gundersen H (1970) Radioimmunoassay for urinary albumin using a single antibody. Scand J Clin Lab Invest 26: 5-11

13. Feldt-Rasmussen B, Dinesen B, Deckert M (1985) Enzyme immunoassay: an improved determination of urinary albumin in diabetics with incipient nephropathy. Scand J Clin Lab Invest 45: 539-544

14. Larsen K (1972) Creatinine assay by a reaction - kinetic principle. Clin Chim Acta 41: 209-217

15. Rossing P, Astrup A-S, Smidt U, Parving H-H (1994) Monitoring kidney function in diabetic nephropathy. Diabetologia 37 : 708-712
16. Bröchner-Mortensen J, Rödbro P (1976) Selection of routine method for determination of glomerular filtration rate in adult patients. Scand J Clin Lab Invest 36: 35-45

17. Mogensen C (1976) Progression of nephropathy in longterm diabetics with proteinuria and effect of initial antihypertensive treatment. Scand J Clin Lab Invest 36: 383-388

18. Parving H-H, Smidt U, Friisberg B, Bonnevie-Nielsen V, Andersen A (1981) A prospective study of glomerular filtration rate and arterial blood pressure in insulin-dependent diabetics with diabetic nephropathy. Diabetologia 20: 457-461

19. Viberti G, Bilous R, Mackintosh D, Keen H (1983) Monitoring glomerular function in diabetic nephropathy. Am J Med 74: 256-264

20. Rossing P (1998) Promotion, prediction, and prevention of progression in diabetic nephropathy. Diabet Med 15: 900-919

21. Kussman M, Goldstein H, Gleason R (1976) The clinical course of diabetic nephropathy. JAMA 236: 1861-1863

22. Mogensen C (1999) Microalbuminuria, blood pressure and diabetic renal disease: origin and development of ideas. Diabetologia 42: 263-285

23. Mogensen C (1982) Long-term antihypertensive treatment inhibiting progression of diabetic nephropathy. BMJ 285: 685-688

24. Parving H-H, Andersen A, Smidt U, Svendsen P (1983) Early aggressive antihypertensive treatment reduces rate of decline in kidney function in diabetic nephropathy. Lancet i: $1175-1179$

25. Lewis E, Hunsicker L, Bain R, Rhode R (1993) The effect of angiotensin-converting-enzyme inhibition on diabetic nephropathy. N Eng J Med 329: 1456-1462

26. Jungers P, Houillier P, Forget D et al. (1995) Influence of pregnancy on the course of primary chronic glomerulonephritis. Lancet 346: 1122-1124

27. Lorenzen T, Pociot F, Johannesen J, Kristiansen O, Nerup J (1999) A population-based survey of frequencies of self-reported spontaneous and induced abortions in Danish women with Type 1 diabetes mellitus. Danish IDDM Epidemiology and Genetics Group. Diabet Med 16: 472-476

28. Miodovnik M, Skillman C, Holroyde J, Butler J, Wendel J, Siddiqi T (1985) Elevated maternal glycohemoglobin in early pregnancy and spontaneous abortion among insulindependent diabetic women. Am J Obstet Gynecol 153: 439-442

29. Damm P, Molsted-Pedersen L (1989) Significant decrease in congenital malformations in newborn infants of an unselected population of diabetic women. Am J Obstet Gynecol 161: 1163-1167

30. Garner P (1995) Type I diabetes mellitus and pregnancy. Lancet 346: 157-161 